PROCEEDINGS OF THE

AMERICAN MATHEMATICAL SOCIETY

Volume 135, Number 9, September 2007, Pages 2821-2826

S 0002-9939(07)08791-6

Article electronically published on February 7, 2007

\title{
EQUILIBRIUM POINTS OF LOGARITHMIC POTENTIALS ON CONVEX DOMAINS
}

\author{
J. K. LANGLEY
}

(Communicated by Juha M. Heinonen)

\begin{abstract}
Let $D$ be a convex domain in $\mathbb{C}$. Let $a_{k}>0$ be summable constants and let $z_{k} \in D$. If the $z_{k}$ converge sufficiently rapidly to $\zeta \in \partial D$ from within an appropriate Stolz angle, then the function $\sum_{k=1}^{\infty} a_{k} /\left(z-z_{k}\right)$ has infinitely many zeros in $D$. An example shows that the hypotheses on the $z_{k}$ are not redundant and that two recently advanced conjectures are false.
\end{abstract}

\section{INTRODUCTION}

A number of recent papers [4, 5, 9, 10, have concerned zeros of functions

$$
f(z)=\sum_{k=1}^{\infty} \frac{a_{k}}{z-z_{k}}
$$

and in particular the following conjecture [4.

Conjecture 1.1 (4]). Let $f$ be given by (11), where $a_{k}>0$ and

$$
z_{k} \in \mathbb{C}, \quad \lim _{k \rightarrow \infty} z_{k}=\infty, \quad \sum_{z_{k} \neq 0}\left|\frac{a_{k}}{z_{k}}\right|<\infty .
$$

Then $f$ has infinitely many zeros in $\mathbb{C}$.

The assumptions of Conjecture 1.1 imply that $f$ is meromorphic in the plane and, assuming that all $z_{k}$ are nonzero, $f(z)$ is the complex conjugate of the gradient of the associated subharmonic potential $u(z)=\sum_{k=1}^{\infty} a_{k} \log \left|1-z / z_{k}\right|$. Moreover, Conjecture 1.1 has a physical interpretation in terms of the existence of equilibrium points of the electrostatic field arising from a system of infinite wires, each carrying a charge density $a_{k}$ and perpendicular to the complex plane at $z_{k}$ [8, p.10]. Conjecture 1.1] is known to be true when $\sum_{\left|z_{k}\right| \leq r} a_{k}=o(\sqrt{r})$ as $r \rightarrow \infty$ [4, Theorem 2.10] (see also [6, p.327]) and when $\inf \left\{a_{k}\right\}>0$ [5] (see also [9]).

An analogue of Conjecture 1.1 for a disc was advanced in [3, Conjecture 2].

Conjecture $1.2([3])$. Let $0<\rho<\infty$ and $\theta \in \mathbb{R}$. Let $f$ be given by (11), where

$$
z_{k} \in \mathbb{C}, \quad\left|z_{k}\right|<\rho, \quad \lim _{k \rightarrow \infty} z_{k}=\rho e^{i \theta}, \quad a_{k}>0, \quad \sum_{k=1}^{\infty} a_{k}<\infty .
$$

Received by the editors February 21, 2006 and, in revised form, May 19, 2006.

2000 Mathematics Subject Classification. Primary 30D35, 31A05, 31B05.

Key words and phrases. Critical points, potentials, zeros of meromorphic functions.

(C)2007 American Mathematical Society Reverts to public domain 28 years from publication 
Then $f$ has infinitely many zeros in $|z|<\rho$.

If $f$ satisfies the assumptions of Conjecture 1.2, then $\bar{f}=\nabla u$ in $|z|<\rho$, where $u(z)=\sum_{k=1}^{\infty} a_{k} \log \left|z-z_{k}\right|$. Obviously there is no loss of generality in assuming that $\rho=1$ and $\theta=0$ in Conjecture 1.2. Write

$$
w=\frac{1}{1-z}, \quad w_{k}=\frac{1}{1-z_{k}}, \quad f(z)=w F(w)
$$

where

$$
F(w)=\sum_{k=1}^{\infty} \frac{a_{k} w_{k}}{w-w_{k}}
$$

It is then easy to verify that Conjecture 1.2 is equivalent to the following statement: if $F$ is given by (5), where

$$
w_{k} \in \mathbb{C}, \quad \operatorname{Re} w_{k}>\frac{1}{2}, \quad \lim _{k \rightarrow \infty} w_{k}=\infty, \quad a_{k}>0, \quad \sum_{k=1}^{\infty} a_{k}<\infty,
$$

then $F$ has infinitely many zeros in $\operatorname{Re} w>1 / 2$.

With the assumptions (6), the function $F$ in (5D) is evidently meromorphic in the plane. In \$2 an example satisfying (5) and (6) will be constructed such that $F$ has no zeros in $\mathbb{C}$. Thus Conjecture 1.2 is false, and there is no direct analogue of Conjecture 1.1 for the unit disc.

On the other hand the following theorem shows in particular that if the $z_{k}$ converge to $\rho e^{i \theta}$ sufficiently rapidly, and if all but finitely many $z_{k}$ lie in a sufficiently small Stolz angle, then the conclusion of Conjecture 1.2 does hold. It is convenient to state and prove the result when the $z_{k}$ lie in a convex domain $D$ and the boundary point $\rho e^{i \theta}$ is 1 . There then exists (see \$4) an open half-plane $H$ such that $D \subseteq H$ and 1 lies on the boundary $\partial H$, and there is no loss of generality in assuming that $H$ is the half-plane $\operatorname{Re} z<1$.

Theorem 1.1. Let $D \subseteq\{z \in \mathbb{C}: \operatorname{Re} z<1\}$ be a convex domain such that $1 \in \partial D$. Let $f$ be given by (11), where

$$
z_{k} \in D, \quad a_{k}>0, \quad \sum_{k=1}^{\infty} a_{k}<\infty .
$$

Assume that 1 is a limit point of the set $\left\{z_{k}: k \in \mathbb{N}\right\}$ and that there exist real numbers $\varepsilon>0$ and $\lambda \geq 0$ such that

$$
\sum_{\left|1-z_{k}\right| \leq \varepsilon}\left|1-z_{k}\right|^{\tau}<\infty \text { for all } \tau>\lambda
$$

and

$$
\sup \left\{\left|\arg \left(1-z_{k}\right)\right|: k \in \mathbb{N},\left|1-z_{k}\right| \leq \varepsilon\right\}<C(\lambda)=\frac{\pi}{2 \lambda} .
$$

Then there exists a sequence $\left(\eta_{j}\right)$ of zeros of $f$ satisfying $\eta_{j} \in D, \lim _{j \rightarrow \infty} \eta_{j}=1$.

Note that (8) implies that $\left\{z_{k}: k \in \mathbb{N}\right\}$ has no limit points $z$ in the punctured $\operatorname{disc} A$ given by $0<|1-z|<\varepsilon$ and that $f$ is meromorphic on $A$. Moreover, (9) is obviously satisfied if $\lambda<1$. 


\section{A counterexample to Conjecture 1.2}

As noted in \$1 it suffices to construct a zero-free function $F$ satisfying (5) and (6). Let

$$
g(w)=\frac{1}{w(w-2)\left(e^{w-1}+1\right)} .
$$

Then $g$ has no zeros, but has simple poles at 0,2 and

$$
u_{k}=1+(2 k+1) \pi i, \quad k \in \mathbb{Z} .
$$

Straightforward computations give

$$
\operatorname{Res}(g, 0)=\frac{-1}{2\left(e^{-1}+1\right)}=-a, \quad \operatorname{Res}(g, 2)=\frac{1}{2(e+1)}=b,
$$

and, using (11),

$$
\operatorname{Res}\left(g, u_{k}\right)=\frac{-1}{u_{k}\left(u_{k}-2\right)}=\frac{-1}{\left(u_{k}-1\right)^{2}-1}=\frac{1}{(2 k+1)^{2} \pi^{2}+1}=c_{k} .
$$

Then $b$ and the $c_{k}$ evidently satisfy

$$
b>0, \quad c_{k}>0, \quad \sum_{k \in \mathbb{Z}} c_{k}<\infty .
$$

Next, let

$$
h(w)=-\frac{a}{w}+\frac{b}{w-2}+\sum_{k \in \mathbb{Z}} \frac{c_{k}}{w-u_{k}}, \quad L(w)=h(w)-g(w) .
$$

By (10), (11), (12), (13) and (14) the function $h(w)$ is meromorphic in the plane, and $L(w)$ is an entire function.

Let $m$ be a large positive integer, let $R=4 m \pi$, and use $c$ to denote positive constants independent of $m$. Then simple estimates give

$$
|g(w)| \leq \frac{c}{R^{2}} \quad \text { for } \quad|w-1|=R
$$

and, as $m \rightarrow \infty$,

$$
|h(w)| \leq \frac{c}{R}+c \sum_{k \in \mathbb{Z},|k| \geq m} c_{k}+c \sum_{k \in \mathbb{Z},|k|<m} \frac{c_{k}}{R}=o(1) \quad \text { for } \quad|w-1|=R .
$$

Combining (16) and (17) shows that $L(w) \equiv 0$ in (15), so that $h=g$ has no zeros, and applying the residue theorem in conjunction with (16) now gives

$$
a=b+\sum_{k \in \mathbb{Z}} c_{k} .
$$

Hence $h(w)$ may be expressed using (18) in the form

$$
\begin{aligned}
h(w) & =b\left(\frac{1}{w-2}-\frac{1}{w}\right)+\sum_{k \in \mathbb{Z}} c_{k}\left(\frac{1}{w-u_{k}}-\frac{1}{w}\right) \\
& =\frac{1}{w}\left(\frac{2 b}{w-2}+\sum_{k \in \mathbb{Z}} \frac{c_{k} u_{k}}{w-u_{k}}\right) .
\end{aligned}
$$

By (11), (14) and (19) the function $F(w)=w h(w)$ may be written in the form

$$
F(w)=\sum_{k=1}^{\infty} \frac{d_{k} v_{k}}{w-v_{k}}, \quad \operatorname{Re} v_{k} \geq 1, \quad v_{k} \rightarrow \infty, \quad d_{k}>0, \quad \sum_{k=1}^{\infty} d_{k}<\infty .
$$


Here $F$ evidently satisfies the requirements of (5) and (6), but $F$ has no zeros in $\mathbb{C}$, since $h$ has no zeros and $h(0)=\infty$.

Remark. It is conjectured further in [3, Conjecture 6] that if $f$ satisfies (11) and (2) with $a_{k} \bar{z}_{k}>0$ for each $k$, then $f$ has infinitely many zeros in $\mathbb{C}$. The example $F$ in (20), with $a_{k}=d_{k} v_{k}$ and $a_{k} \bar{v}_{k}=d_{k}\left|v_{k}\right|^{2}>0$, shows that this conjecture is also false. Moreover, the example $h$ in (15) satisfies all the conditions of Conjecture 1.1 except that $\operatorname{Res}(h, 0)<0$. Thus a single negative charge may invalidate the conclusion of Conjecture 1.1

\section{An AuXiliary ReSUlt NEEded For TheOrem 1.1}

The proof of Theorem 1.1 rests upon the following proposition, which concerns functions of the form (5) and uses standard notation from [7, p.42]. For a related result, see [9, Theorem 1.6].

Proposition 3.1. Let $0<\sigma \leq 1$. Let $F$ be given by (5), where

$$
w_{k} \in \mathbb{C}, \quad \operatorname{Re} w_{k}>0, \quad a_{k}>0, \quad \sum_{k=1}^{\infty} a_{k}<\infty .
$$

Assume that the set $\left\{w_{k}: k \in \mathbb{N}\right\}$ is unbounded and that there exist real numbers $R>0$ and $\lambda \geq 0$ such that

$$
\sum_{\left|w_{k}\right| \geq R}\left|w_{k}\right|^{-\tau}<\infty \text { for all } \tau>\lambda
$$

and

$$
s=\sup \left\{\left|\arg w_{k}\right|: k \in \mathbb{N},\left|w_{k}\right| \geq R\right\}<C(\lambda, \sigma)=\frac{2}{\lambda} \arcsin \sqrt{\frac{\sigma}{2}} .
$$

Then there exists a function $G$, transcendental and meromorphic in the plane, with

$$
F(w)=G(w)(1+o(1)) \quad \text { as } \quad w \rightarrow \infty
$$

and the Nevanlinna deficiency $\delta(0, G)$ of the zeros of $G$ satisfies $\delta(0, G)<\sigma$. In particular, $F(w)$ has a sequence of zeros tending to infinity.

The zero-free example of (20) has $\lambda=1$ and $\delta(0, F)=\sigma=1$, and all its poles lie in $\operatorname{Re} w \geq 1$, so that Proposition 3.1 is essentially sharp.

To prove Proposition 3.1, assume that $F$ is as in the statement of Proposition 3.1. It follows from (22) that the set $\left\{w_{k}: k \in \mathbb{N}\right\}$ has no limit points $w$ with $R<|w|<\infty$. In particular, $F$ is meromorphic in the region $2 R \leq|w|<\infty$ with an essential singularity at infinity. The existence of a transcendental meromorphic function $G$ satisfying (24) then follows from a result of Valiron [12, p.15] (see also [2, p.89]). In particular, $G$ is constructed [12] so that $F$ and $G$ have the same poles and zeros in $|w| \geq 2 R$. If $|w| \geq 4 R$, then (21) gives

$$
|F(w)| \leq\left|F_{1}(w)\right|+O(1), \quad F_{1}(w)=\sum_{\left|w_{k}\right| \geq 2 R} \frac{a_{k} w_{k}}{w-w_{k}},
$$

so that

$$
m(r, G) \leq m\left(r, F_{1}\right)+O(1)=O(1)
$$

as $r \rightarrow \infty$, by [6, p.327]. Since the poles $w_{k}$ of $G$ have exponent of convergence at most $\lambda$ by (22), it follows that $G$ has lower order $\mu \leq \lambda$. 
Choose $s_{0}, s_{1}, s_{2}$ with

$$
s<s_{0}<s_{1}<s_{2}<\min \{\pi, C(\lambda, \sigma)\},
$$

where $s$ is as in (23) and satisfies $s \leq \pi / 2$ by (21). The proof of Proposition 3.1 requires the following two lemmas.

Lemma 3.1. The function $F$ satisfies $\liminf _{r \in \mathbb{R}, r \rightarrow+\infty} r|F(-r)|>0$.

Proof. Let $r>0$ and write $w_{k}=u_{k}+i v_{k}$ with $u_{k}$ and $v_{k}$ real. Let

$$
p_{k}(r)=\operatorname{Re}\left(\frac{w_{k}}{r+w_{k}}\right)=\frac{u_{k}\left(r+u_{k}\right)+v_{k}^{2}}{\left(r+u_{k}\right)^{2}+v_{k}^{2}} .
$$

Then (21) gives $p_{k}(r)>0$, and there exists $d>0$ such that $p_{1}(r)>d / r$ as $r \rightarrow \infty$. Hence, again as $r \rightarrow \infty$,

$$
r|F(-r)| \geq-r \operatorname{Re} F(-r)=r \sum_{k=1}^{\infty} a_{k} p_{k}(r) \geq r a_{1} p_{1}(r)>a_{1} d .
$$

Lemma 3.2. There exists $M_{1}>1$ such that $|F(w)| \leq M_{1}$ for all large $w$ lying outside the region $|\arg w|<s_{0}$.

Proof. This follows from (21), (23) and (25), since there exists a positive constant $M_{2}$ such that $\left|w-w_{k}\right| \geq M_{2}\left|w_{k}\right|$ for all such $w$ and all $k \in \mathbb{N}$.

The proof of Proposition 3.1 may now be completed using Lemmas 3.1 and 3.2 . Assume that $\delta(0, G) \geq \sigma$. Then Baernstein's spread theorem [1] gives a sequence $r_{m} \rightarrow \infty$ and, for each $m$, a subset $I_{m}$ of the circle $|w|=r_{m}$, of angular measure at least

$$
\min \left\{2 \pi, \frac{4}{\mu} \arcsin \sqrt{\frac{\sigma}{2}}\right\}-o(1) \geq \min \{2 \pi, 2 C(\lambda, \sigma)\}-o(1) \geq 2 s_{2},
$$

using (23) and (25), and such that

$$
\lim _{m \rightarrow \infty} L_{m}=-\infty, \quad \text { where } \quad L_{m}=\frac{\max \left\{\log |G(w)|: w \in I_{m}\right\}}{\log r_{m}} .
$$

Let $m$ be large, and consider the function $v(w)=\log |F(w)|$, which is subharmonic on the domain

$$
\Omega=\left\{w \in \mathbb{C}: r_{m} / 4<|w|<r_{m}, s_{0}<\arg w<2 \pi-s_{0}\right\} .
$$

Then $v$ is bounded above on $\Omega$, by Lemma 3.2. But the intersection $J_{m}$ of $I_{m}$ with the arc $\left\{w \in \mathbb{C}:|w|=r_{m}, s_{1}<\arg z<2 \pi-s_{1}\right\}$ has angular measure at least $2\left(s_{2}-s_{1}\right)$, so that standard estimates for the harmonic measure of $J_{m}$ at $-r_{m} / 2$ now give

$$
\omega\left(-r_{m} / 2, J_{m}, \Omega\right) \geq M_{3}>0,
$$

where $M_{3}$ is independent of $m$. Combining (24), (26), (27) and Lemma 3.2 and applying the two-constants theorem [11, p.42] to $v$ leads to, as $m \rightarrow \infty$,

$$
v\left(-r_{m} / 2\right) \leq M_{3} L_{m} \log r_{m}+\log M_{1}+o(1), \quad r_{m} F\left(-r_{m} / 2\right) \rightarrow 0 .
$$

But (28) contradicts Lemma 3.1, and this completes the proof of Proposition 3.1, 


\section{Proof of Theorem 1.1}

Assume that $f$ and $D$ satisfy the hypotheses of Theorem 1.1. Define $F$ using the transformations (4) and (5). Then $F$ satisfies the hypotheses of Proposition 3.1 with $R=1 / \varepsilon$ and $\sigma=1$. Thus $F$ has a sequence of zeros tending to infinity, and so $f$ has a sequence $\left(\eta_{j}\right)$ of zeros with $\lim _{j \rightarrow \infty} \eta_{j}=1$.

It remains only to show that such a sequence $\left(\eta_{j}\right)$ exists with, in addition, $\eta_{j} \in D$, and this is done by a standard argument of Gauss-Lucas type. Let $\eta=\eta_{j}$ with $j$ large, and assume that $\eta \notin D$. Since $D$ is convex the supremum and infimum of $\arg (z-\eta)$ on $D$ differ by at most $\pi$. Hence there exist an open half-plane $H$, with $D \subseteq H$ and $\eta \in \partial H$, and a linear transformation $u=T(z)=(z-\eta) / a$ mapping $H$ onto $\operatorname{Re} u>0$. Writing $u_{k}=T\left(z_{k}\right)$ then gives

$$
0=\operatorname{Re}(a f(\eta))=-\operatorname{Re}\left(\sum_{k=1}^{\infty} \frac{a_{k}}{u_{k}}\right)<0 .
$$

This contradiction completes the proof of Theorem 1.1

\section{REFERENCES}

[1] A. Baernstein, Proof of Edrei's spread conjecture, Proc. London Math. Soc. (3) 26 (1973), 418-434. MR0374429(51:10629)

[2] L. Bieberbach, Theorie der gewöhnlichen Differentialgleichungen, 2. Auflage, Springer, Berlin, 1965. MR0176133 (31:408)

[3] J. Borcea, Equilibrium points of logarithmic potentials induced by positive charge distributions I: generalised de Bruijn-Springer relations, Trans. Amer. Math. Soc., to appear.

[4] J. Clunie, A. Eremenko and J. Rossi, On equilibrium points of logarithmic and Newtonian potentials, J. London Math. Soc. (2) 47 (1993), 309-320. MR1207951 (94c:31001)

[5] A. Eremenko, J.K. Langley and J. Rossi, On the zeros of meromorphic functions of the form $\sum_{k=1}^{\infty} \frac{a_{k}}{z-z_{k}}$, J. d'Analyse Math. 62 (1994), 271-286. MR.1269209(95f:30041)

[6] A.A. Gol'dberg and I.V. Ostrovskii, Distribution of values of meromorphic functions, Nauka, Moscow 1970. MR0280720(43:6439)

[7] W.K. Hayman, Meromorphic functions, Oxford at the Clarendon Press, 1964.

[8] O.D. Kellogg, Foundations of potential theory, Springer, Berlin, 1967. MR0164038 (29:1337)

[9] J.K. Langley and J. Rossi, Meromorphic functions of the form $f(z)=\sum_{n=1}^{\infty} a_{n} /\left(z-z_{n}\right)$, Rev Mat. Iberoamericana 20 (2004), 285-314. MR2076782 (2005d:30046)

[10] J.K. Langley and John Rossi, Critical points of certain discrete potentials, Complex Variables 49 (2004), 621-637. MR2088052 (2005f:30059)

[11] R. Nevanlinna, Eindeutige analytische Funktionen, 2. Auflage, Springer, Berlin, 1953. MR0057330 (15:208c)

[12] G. Valiron, Lectures on the general theory of integral functions, Chelsea, New York, 1949.

School of Mathematical Sciences, University of Nottingham, NG7 2RD, United KingDOM

E-mail address: jkl@maths.nott.ac.uk 